\title{
Comparison of Kyphoplasty and Lordoplasty in the Treatment of Osteoporotic Vertebral Compression Fracture
}

\author{
Sang-Bum Kim¹, Taek-Soo Jeon², Woo-Suk Lee', \\ Jae-Young Roh', Jae-Young Kim¹, Won-Ki Park' \\ 'Department of Orthopaedic Surgery, Konyang University College of Medicine, Daejeon, Korea \\ ${ }^{2}$ Department of Orthopaedic Surgery, Haeundae Hospital, Inje University College of Medicine, Busan, Korea
}

Study Design: A retrospective study.

Purpose: To compare the level of restoration of the vertebral height, improvement in the wedge and kyphotic angles, and the incidence of complications in osteoporotic compression fracture in patients treated with either kyphoplasty or lordoplasty.

Overview of Literature: Kyphoplasty involves recompression of the vertebral bodies. Recently, a more effective method known as lordoplasty was introduced.

Methods: Between 2004 and 2009, patients with osteoporotic thoracolumbar vertebral compression fractures were treated by either kyphoplasty $(n=24)$ or lordoplasty $(n=12)$ using polymethylmethacrylate (PMMA) cement, and the results of the two interventions were compared. A visual analogue scale was used to measure the pain status. Preoperative and postoperative radiographs were analyzed to quantify the anterior vertebral height restoration and the wedge and kyphotic alignment correction.

Results: All patients in both groups reported a significant decrease in pain. The anterior heights increased $24.2 \%$ and $17.5 \%$ after the lordoplasty and kyphoplasty procedures, respectively $(p<0.05)$. Three months after the procedures, there was a larger decrease in the loss of anterior vertebral height in the kyphoplasty group (12.8\%) than in the lordoplasty group $(6.3 \%$, $p<0.05)$. The wedge angles decreased after both procedures. The wedge angle in the lordoplasty group maintained its value after 3 months $\left(p<0.05\right.$. The kyphotic angular correction was 11.4 and $7.0^{\circ}$ in the lordoplasty and kyphoplasty groups, respectively $\left(p<0.05\right.$ ). Both kyphotic deformities worsened to a similar degree of $5^{\circ}$ after 3 months.

Conclusions: Lordoplasty is more useful than kyphoplasty in terms of the improved anatomic restoration and postoperative maintenance.

Key Words: Osteoporotic vertebral compression fracture, Lordoplasty, Vertebroplasty

\section{Introduction}

Osteoporosis is an age-related progressive disease of the skeletal system involving a loss of bone mass, microarchi- tectural deterioration, and an increased susceptibility to bone fractures. Fractures of the vertebral bodies are the most common osteoporotic fractures, and often express the debilitating features of osteoporosis [1]. All vertebral compression fractures $(\mathrm{VCF})$ are associated with increased mor-

Received Nov 22, 2009; 1st Revised Jan 4, 2010; 2nd Revised Jan 26, 2010; 3rd Revised Feb 2, 2010; Accepted Feb 3 , 2010

Corresponding author: Sang-Bum Kim, MD

Department of Orthopaedic Surgery, Konyang University Hospital,

685 Gasuwon-dong, Seo-gu, Daejeon 302-718, Korea

Tel: +82-42-600-6902, 9120, Fax:+82-42-545-2373, E-mail: sbkim@kyuh.co.kr 
tality and morbidity [2]. The progressive loss of posture as a result of this fracture is one of the major problems associated with its enormous impact on the quality of life. A vertebral deformity leads to the relapse of pain, cosmetic problems, decreased activity, change in mood, and reduced pulmonary function, which subsequently results in a decrease in thoracic volume and an increased risk of further fractures of the adjacent vertebra [3,4].

Vertebroplasty (VP) and kyphoplasty (KP) are two minimally invasive surgical procedures that can be used to treat osteoporotic VCF. It is clear that cement augmentation with VP or KP stabilizes the fractured vertebra, offers immediate pain relief and improves the quality of life $[5,6]$.

Despite the high success with the VP procedure, VP does not address the issues of vertebral height loss and kyphotic deformity. KP was designed to achieve a more favorable angle of kyphosis and assist in realigning the spine [7]. Although KP can increase the height of a fractured vertebra body significantly and reduce its kyphotic angle, it still has the characteristics of the recompression of vertebral bodies and carries the risk of subsequent VCF [8,9].

Recently, a more effective method, lordoplasty (LP), was introduced by Orler et al. [10]. The vertebral body above and below the fractured one are reinforced bilaterally as fragile adjacent vertebra. The cannulas are used as a lever, and a lordotic moment is applied to reduce the fractured vertebra body. No study has compared LP and KP directly. Therefore, this study examined and compared the effects of LP and KP procedures on the radiographic parameters and complications.

\section{Materials and Methods}

This retrospective clinical and radiologic review was performed on all consecutive patients who had undergone KP or LP at the orthopedic department of the Konyang University Hospital from 2004 to 2009. The patients suffered from a fracture that was either severely disabling or persistently symptomatic, which was unresponsive to nonsurgical treatment. Nonsurgical treatment consisted of immobilization, brace and medication for at least 2 weeks. Magnetic resonance imaging studies were obtained and reviewed to assess the level of marrow replacement with edema in the fractured vertebra. This study examined patients 60 years of age and older. KP and LP was carried out on patients with a fresh thoracic or lumbar, single VCF not involving a neurologic deficit, and compression fractures type $\mathrm{A} 1$ and $\mathrm{A} 2$ according to Magerl's classification. Patients with multiple segment fractures or old adjacent-level fractures were excluded. Patients with pathological fractures in patients with a neoplasm were also excluded. The T-scores in the bone mineral densities of all patients were <-3.0.

The study population consisted of 12 and 24 patients who had undergone LP and KP, respectively. In the KP group, there were 21 women and 3 men, ranging in age between 60 and 78 years (mean age, 70.8 years). The locations and numbers of the treated vertebral bodies were as follows: T11 ( $=6)$, T12 ( $=4)$, and L1 $(n=2)$. In the LP group, there were 12 women ranging age between 60 and 87 years (mean age, 74.3 years). The locations and number of treated vertebral bodies are as follows: T8 $(n=2)$, T9 $(n=3)$, T10 $(\mathrm{n}=1)$, T11 $(\mathrm{n}=3), \mathrm{T} 12(\mathrm{n}=8), \mathrm{L} 1(\mathrm{n}=3), \mathrm{L} 2(\mathrm{n}=3)$, and L3 $(\mathrm{n}=1)($ Table 1$)$.

\section{Surgical technique}

Under spinal anesthesia, all patients treated with LP were placed in hyperextension to support a restoration. The anesthetist was consulted when high-level anesthesia was required. The abdomen of the patient was allowed to hang freely with a pad supporting the pelvis and sternum. A stab incision was made on the pedicle level of the skin. The correct incision site was indentified with the anteroposterior (AP) view of the image intensifier. A guide wire was placed via a stab incision, and the position of the tip of wire was cranial and lateral of the pedicle projection. The wire was led using long forceps to keep the operator's hand away from the X-ray path. The guide wire was then penetrated further with hammer blows until the tip reached the medial limitation of the pedicle. This step was repeated on another pedicle of the fractured vertebra and adjacent upper and lower vertebrae. In this event, six Kirschner wires were used to perform LP of one fractured vertebra. The depth of the tip of the wire was verified on the lateral view of the image intensifier. It is important that the wire tip be at least over the posterior cortex of the vertebral body. The filling cannula was then pushed over the wire by rotating movements. The tip of the cannula must also be over the ventral half of the vertebral body. The guide wire was removed and the bony remnants in the cannula were cleared with a blunt trochar. A blunt trochar should be inserted into the anterior border of the vertebral body to ensure a pathway for the cement. The cement was mixed and placed into a $20 \mathrm{ml}$ syringe, which was then transferred to $3 \mathrm{ml}$ and $1 \mathrm{ml}$ 

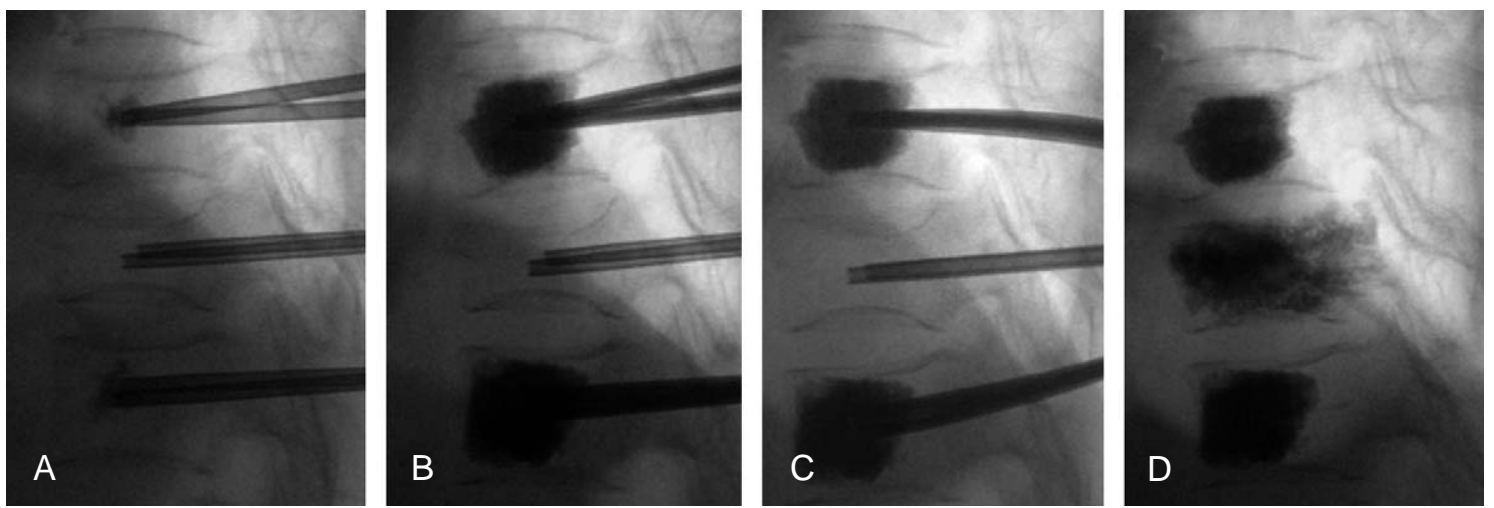

Fig. 1. The procedures of loroplasty. (A) The cement was injected under control with an image intensifier. The condition of the cement at the tip of the cannula must be observed. (B) The cement spread like a growing cloud and should be injected gradually. (C) Relevant decrease in the vertebral body was achieved with a lordotic force. (D) Lordoplasty was completed.

syringes. The cement should be highly viscous before it is injected and not be dropped from the syringe. All cannulas were placed, as described above, and both the distal and proximal vertebral body was augmented first. The cannula was filled carefully with cement in a $1.5 \mathrm{ml}$ syringe. As soon as the cement was beaded at the tip of cannula, the next cannula was filled with the same procedure (Fig. 1A). The filling was carried out separately to control the cannulas. The filling was always carried out under lateral control with an image intensifier. After filling the cannula, the cement was injected into the vertebral body through a $1 \mathrm{ml}$ syringe. The spread of the cement was monitored by lateral control with an image intensifier (Fig. 1B). One to two $\mathrm{ml}$ of cement was sufficient for each cannula. After the cement had hardened, a lordosing force was applied via the cannula in the place (Fig. 1C). The fracture of vertebral body was reduced in the sense of ligamentotaxis using adjacent cemented vertebrae as levers. The reduction force of cannula was held with a cross bolt. In other words, the anterior height was recovered by cantilever bending. Being kept in a reduced state by a cross bolt, the fractured vertebra body was augmented using another activated cement. The lordosing force was not loosened until the cement had hardened. After the cement had hardened, the cannula was removed with a slight turn (Fig. 1D).

All KP procedures were performed with local anesthesia under moderate sedation. The interventions were performed in an operating room with the patient lying prone. KP was also performed through a bipedicular approach using standard KP equipment Kyphon $^{\mathrm{TM}}$, Sunnyvale, CA, USA). Frequent AP and lateral fluoroscopic images were used to confirm the position. Through a working cannula, a drill was
Table 1. The basic characteristics of study group

\begin{tabular}{lccc}
\hline \hline & Total & Lordoplasty & Kyphopalsty \\
\hline No. of cases & 36 & 12 & 24 \\
Mean age $(y r)$ & 73.3 & 78.6 & 70.2 \\
M : F & $3: 33$ & $0: 12$ & $3: 21$ \\
BMD (T-score) & -3.71 & -3.83 & -3.65 \\
Location & & T11-L1 & T8-L3 \\
\hline
\end{tabular}

M: Male, F: Female, BMD: Bone mineral density.

used to create a path for the balloon into the center of the vertebral body. Once inserted, the balloons were inflated until either the fracture was reduced or the inflation pressure reached 220 psi. Polymethylmethacrylate was then injected into the void cavity.

\section{Outcome assessment}

The patient's outcomes were assessed using self-report and physiological measures. Each patient underwent standardized examinations three times: a preoperative examination and radiographic evaluation performed on the day before surgery, the 1st day after surgery and 3 months later. The patients recorded their own assessment of back pain on a visual analogue scale (VAS; $0=$ no pain, $10=$ intolerable pain) and radiographs were taken three times. Dual-energy $\mathrm{X}$-ray absorptiometry scans were recommended for all 36 patients.

The radiographs were obtained and analyzed to quantify the vertebral and local kyphosis correction. AP and lateral radiographs were used to measure the sagittal angles. Preoperative and postoperative vertebral heights at the fractured and adjacent level were measured in the anterior por- 
Table 2. Anterior height, wedge angle and kyphotic angle

\begin{tabular}{llcrr}
\hline \hline & & Preoperative & Postoperative & 3 months \\
\hline Anterior height $(\%)$ & Lordoplasty & $64.7 \pm 3.6$ & $88.8 \pm 2.9$ & $82.5 \pm 4.5$ \\
& Kyphopalsty & $68.7 \pm 1.4$ & $86.2 \pm 1.1$ & $73.4 \pm 1.3$ \\
Wedge angle $\left({ }^{\circ}\right)$ & Lordoplasty & $16.2 \pm 1.0$ & $8.3 \pm 1.2$ & $8.4 \pm 0.9$ \\
& Kyphopalsty & $12.9 \pm 1.3$ & $6.8 \pm 1.2$ & $11.0 \pm 1.2$ \\
Kyphotic angle $\left({ }^{\circ}\right)$ & Lordoplasty & $25.0 \pm 3.3$ & $13.6 \pm 2.3$ & $18.9 \pm 3.0$ \\
& Kyphopalsty & $18.1 \pm 2.6$ & $12.2 \pm 1.6$ & $15.0 \pm 1.8$ \\
\hline
\end{tabular}

Table 3. The change of radiologic parameters

\begin{tabular}{|c|c|c|c|}
\hline & & Restoration & Loss \\
\hline \multirow[t]{3}{*}{ Anterior height (\%) } & Lordoplasty & $24.2 \pm 3.5$ & $6.3 \pm 2.6$ \\
\hline & Kyphopalsty & $17.5 \pm 1.4$ & $12.8 \pm 1.0$ \\
\hline & $p$-value & 0.042 & 0.04 \\
\hline \multirow[t]{3}{*}{ Wedge angle $\left(^{\circ}\right)$} & Lordoplasty & $8.0 \pm 1.0$ & $0.1 \pm 0.5$ \\
\hline & Kyphopalsty & $5.8 \pm 1.0$ & $4.2 \pm 0.5$ \\
\hline & $p$-value & 0.119 & $<0.001$ \\
\hline \multirow[t]{3}{*}{ Kyphotic angle $\left({ }^{\circ}\right)$} & Lordoplasty & $11.4 \pm 1.3$ & $5.3 \pm 1.0$ \\
\hline & Kyphopalsty & $7.0 \pm 1.3$ & $4.4 \pm 0.5$ \\
\hline & $p$-value & 0.392 & 0.043 \\
\hline
\end{tabular}

tions (Table 2). The absolute values of the three vertical bodies (the fractured vertebra with the vertebra above and below) were compared on plain lateral radiographs. The correction of the deformity caused by the fracture was determined from the angle of kyphosis and ascertained by measuring the angle between the superior and inferior endplates on the lateral X-ray images on the fractured vertebra (Table 2). The local kyphotic angle were measured at the cephalad and caudal endplates of the vertebra as well as above and below. Any extravasation of cement, adjacent vertebral fracture and recompression of fractured vertebra were also assessed on the plain X-ray films.

\section{Statistical analysis}

Statistical analysis was performed using commercially available software (SPSS ver. 12.0; SPSS Inc., Chicago, IL, USA). The statistical significance of the changes in vertebral height, wedge angle and local kyphosis with each treatment were evaluated using a paired $t$-test. The statistical significance of the changes in vertebral height, wedge angle and local kyphosis with two treatment techniques were evaluated using an independent $t$-test. Comparisons of the complications of both two groups were evaluated using a Fischer's exact test. A $p$-value $<0.05$ was considered significant.

\section{Results}

All patients reported a significant decrease in pain within 24 hours postoperatively. The back pain recorded using the VAS (range, 0-10) in the LP group improved significantly in all cases, from $9.0 \pm 0.6$ (range, 8-10) points preoperatively to $2.1 \pm 0.8$ (range, 1-3) points postoperatively, and then to $2.2 \pm 0.8$ (range, 1-3) points at the follow-up. In the KP group, the severity of back pain also decreased significantly, from $8.8 \pm 0.6$ (range, 8 -10) points preoperatively to $2.2 \pm 0.8$ (range, 1-3) points postoperatively and then to $1.9 \pm 0.3$ (range, 1-2) points at the follow-up. There was no detectable difference in pain relief between the two groups $(p=0.332)$.

The anterior heights increased $24.2 \pm 3.5$ and $17.5 \pm$ $1.4 \%$ after the LP and KP procedures, respectively ( $p=$ 0.042). The decrease in the loss of anterior vertebral height was larger for the KP group than the LP group at 3 months after the procedures $(p=0.040)$. The wedge angles decreased after both procedures $(p=0.019)$. However, after 3 months, only the wedge angle in LP group was maintained $(p<0.001)$. The kyphotic angular correction was 11.4 and $7.0^{\circ}$ after the LP and KP procedures, respectively $(p=0.023)$. Both kyphotic deformities became worse to a similar degree $\left(5^{\circ}\right)$ after 3 months $(p=0.354)$ (Table 3$)$. 
Cement leakage outside the vertebral body was observed in 6 out of 12 vertebrae (50\%) treated with LP and in 7 out of 26 vertebrae (26.9\%) treated with KP. There was no significant cement extravasation into the canal or foramina and no emboli observed during the two procedures. Seven patients in the KP group suffered a subsequent vertebral compression fracture at the 12-week follow up. All subsequent fractures were treated conservatively. A comparison of the rates of cement leakage $(p=0.332)$ and adjacent vertebral fracture $(p=0.069)$ showed no significant differences between the two groups. There were no additional fractures in the adjacent cemented vertebrae for LP on the postoperative computed tomogram.

\section{Discussion}

LP was first introduced by Professor Orler et al. [10] as an alternative to KP in 2006. VP simply introduces cement, and a correction of the vertebral structure is occasionally achieved. KP might achieve the correction to a certain extent but it has many problems. In an analogy with the principles of internal fixation, indirect repositioning is achieved with the support of the adjoining vertebrae via ligamentotaxis. Unlike KP, where the initial reduction can frequently disappear after deflating the balloons, the fractured vertebra can be augmented as a consequence of the existing prestressing in LP.

There are no articles comparing the results of LP and KP directly. This paper reports that both LP and KP lead to a significant decrease in fracture pain. It was also shown that anatomic restoration can be improved successfully using both procedures, even though LP has a more advantageous degree of restoration and maintenance.

Previous papers on KP reported a $4-10^{\circ}$ decrease in kyphotic angle and a meaningful restoration of the vertebral height after the procedure [11-13]. Heini and Orler [6] performed the technique using the lordotic moment to reduce the fractured vertebra. They reported $15.2^{\circ}$ and $10^{\circ}$ improvement in the compressed wedge angle and kyphotic angle, respectively, at the 8 week follow-up after the LP procedure [10].

In this study, the vertebral wedge angle and kyphotic angle were restored postoperatively by approximately $8.0^{\circ}$ and $11.4^{\circ}$, respectively. The LP group showed significant anatomic restoration of the fractured vertebra in terms of the anterior vertebral height as well as the vertebral wedge and kyphotic angles compared to KP. The rate of re-col- lapse after the procedures in the KP group was significantly higher than that in the LP group.

Recollapse of the vertebral body after KP leads to a change in local kyphosis, back pain, and the risk of an adjacent vertebral fracture. Several studies reported the realignment of KP $[9,14,15]$. At the center of the upper end plate, a biomechanical evaluation revealed greater subsidence in the KP group than in the VP group [8]. The percentage of the intact height of the vertebral bodies treated with KP is larger than that with VP, but smaller after cyclic loading [16]. In the LP group, the subsidence decreased because the lordotic movement is given by VP, which helps maintain a constant kyphotic angle in the long term.

Vertebral body fractures in the adjacent vertebra after VP or KP are induced by an anterior shift of the upper body $[15,17]$. However, it is unclear how a wedge shaped compression fracture of a vertebra increases both the forces of the trunk muscle and intradiscal pressure in the adjacent discs [17]. The advantage of KP reported in Rohlmann's study is apparent only if almost full fracture reduction is achieved. Finite element models have shown that an adjacent vertebra fracture may result from an altered load distribution called a pillar of cement. A pillar of cement increases the pressure in the adjacent nucleus pulposus and endplate of the adjacent vertebra [18].

To the best of our knowledge, there are few reports on prophylactic therapy on the adjacent vertebra after VP. Although prophylactic cement augmentation is controversial, prophylactic cement augmentation stabilizes the osteoporotic vertebra and reduces the spinal deformity [19]. The risk of a new fracture in the upper adjacent vertebra will be increased because the intervertebral disc adjacent to the superior end plate of a fractured vertebra is often degenerated from the decreased buffer action of the cartilaginous component $[20,21]$. Therefore, a prophylactic cement injection into the vertebra immediately above the fractured vertebra may be justified [22]. Chiang et al. [23] reported the advantages of prophylactic cement augmentation, such as a decreased risk of failure of the augmented vertebra maintaining an intact adjacent non-augmented vertebra from a fatigue injury. Kobayashi et al. [22] recently compared 89 cases in a non-prophylactic group with 155 cases in a prophylactic group and showed that fewer new fractures developed in the prophylactic group. In the present study, no adjacent vertebral fracture was observed despite the rather small number of cases. Orler et al. [10] also reported only one case in 36 cases. In the LP procedure, preventive 
administration is automatically performed on an adjacent vertebral fracture by VP treatment. Pressure transfer to the adjacent segment appears to be minimized in the LP case because complete restoration of the anatomic shape is possible and the curing effect of the kyphotic angle is remarkable.

Adverse effects are the major concern when a lordotic moment to reduce the fractured vertebra and multiple-level VP inducing prophylactic treatment are performed. A lordotic force carries the risk of unsuspected damage to the adjacent vertebral pedicle and facet joints. The risk of latent complications, such as cement leakage and pulmonary embolism, may increase with increasing number of treated vertebra $[24,25]$. In the present study, computed-tomography showed no injury to the adjacent vertebral structures after LP on the postoperative scan. The cemented vertebral body acts as a lever, which can eliminate damage to the adjacent vertebral pedicle and facet joints. The relationship between the number of vertebra treated and the risk of complications could not be clarified [22].

A systemic review of clinical studies reported that the rate of cement leakage was $9 \%$ and $41 \%$ after KP and VP, respectively [26]. An up-to-date meta-analysis reported cement extravasation in $7 \%$ and $20 \%$ of patients after KP and VP, respectively [27]. Our result of 50\% in the LP group exceeded these values. The use of high viscosity cement and a large diameter cannula might decrease the risk of cement leakage. In this study, cement leakage did not cause clinical symptoms or affect the treatment outcome.

The cost associated with each procedure is also an important factor. The excessive cost and more complex procedure of KP compared with VP places in question the clinical value of KP. LP is more cost-effective than KP. In the republic of Korea, KP costs US\$3,644, whereas LP costs US\$1,525.

This study had some limitations. First, this study was not a prospective randomized trial. The KP group was studied first, followed by LP. LP was studied prospectively and KP was examined retrospectively. The small number of cases is a second limitation. Therefore, studies with a larger number of patients will be needed. Third, even if most adjacent vertebral fractures had occurred in the two months after the procedures, a longer follow-up will be needed to clearly evaluate the other complications. Fourth, the vertebra of only the thoracolumbar junction was treated mainly by LP. Fifth, the effect of the vertebral body above and below the prophylactic augmented ones was not examined. Overall, further studies including larger number of patients and a longer follow-up period will be needed to evaluate the clinical efficacy and adverse effects of LP.

\section{Conclusions}

LP is more useful than KP in terms of the increased anatomic restoration, increased postoperative maintenance and cost-effectiveness.

\section{REFERENCES}

1. Melton LJ 3rd. Epidemiology of spinal osteoporosis. Spine (Phila Pa 1976) 1997;22(24 Suppl):2S-11S.

2. Dreinhöfer KE, Féron JM, Herrera A, et al. Orthopaedic surgeons and fragility fractures: a survey by the Bone and Joint Decade and the International Osteoporosis Foundation. J Bone Joint Surg Br 2004;86:958-61.

3. Burger H, Van Daele PL, Grashuis K, et al. Vertebral deformities and functional impairment in men and women. J Bone Miner Res 1997;12:152-7.

4. Schlaich C, Minne HW, Bruckner T, et al. Reduced pulmonary function in patients with spinal osteoporotic fractures. Osteoporos Int 1998;8:261-7.

5. Ploeg WT, Veldhuizen AG, The B, Sietsma MS. Percutaneous vertebroplasty as a treatment for osteoporotic vertebral compression fractures: a systematic review. Eur Spine J 2006;15:1749-58.

6. Heini PF, Orler R. Kyphoplasty for treatment of osteoporotic vertebral fractures. Eur Spine J 2004;13:184-92.

7. Garfin SR, Yuan HA, Reiley MA. New technologies in spine: kyphoplasty and vertebroplasty for the treatment of painful osteoporotic compression fractures. Spine (Phila Pa 1976) 2001;26:1511-5.

8. Wilke HJ, Mehnert U, Claes LE, Bierschneider MM, Jaksche H, Boszczyk BM. Biomechanical evaluation of vertebroplasty and kyphoplasty with polymethyl methacrylate or calcium phosphate cement under cyclic loading. Spine (Phila Pa 1976) 2006;31:2934-41

9. Fribourg D, Tang C, Sra P, Delamarter R, Bae H. Incidence of subsequent vertebral fracture after kyphoplasty. Spine (Phila Pa 1976) 2004;29:2270-6.

10. Orler R, Frauchiger LH, Lange U, Heini PF. Lordoplasty: report on early results with a new technique for the treatment of vertebral compression fractures to restore the lordosis. Eur Spine J 2006;15:1769-75.

11. Liu JT, Liao WJ, Tan WC, et al. Balloon kyphoplasty ver- 
sus vertebroplasty for treatment of osteoporotic vertebral compression fracture: a prospective, comparative, and randomized clinical study. Osteoporos Int 2010;21:359-64.

12. Theodorou DJ, Theodorou SJ, Duncan TD, Garfin SR, Wong WH. Percutaneous balloon kyphoplasty for the correction of spinal deformity in painful vertebral body compression fractures. Clin Imaging 2002;26:1-5.

13. Fourney DR, Schomer DF, Nader R, et al. Percutaneous vertebroplasty and kyphoplasty for painful vertebral body fractures in cancer patients. J Neurosurg 2003;98(1 Suppl):21-30.

14. Lavelle WF, Cheney R. Recurrent fracture after vertebral kyphoplasty. Spine J 2006;6:488-93.

15. Harrop JS, Prpa B, Reinhardt MK, Lieberman I. Primary and secondary osteoporosis' incidence of subsequent vertebral compression fractures after kyphoplasty. Spine (Phila Pa 1976) 2004;29:2120-5.

16. Kim MJ, Lindsey DP, Hannibal M, Alamin TF. Vertebroplasty versus kyphoplasty: biomechanical behavior under repetitive loading conditions. Spine (Phila Pa 1976) 2006;31:2079-84.

17. Rohlmann A, Zander T, Bergmann G. Spinal loads after osteoporotic vertebral fractures treated by vertebroplasty or kyphoplasty. Eur Spine J 2006;15:1255-64.

18. Wang JL, Wu TK, Lin TC, Cheng CH, Huang SC. Rest cannot always recover the dynamic properties of fatigueloaded intervertebral disc. Spine (Phila Pa 1976) 2008;33:1863-9.

19. Yuan HA, Brown CW, Phillips FM. Osteoporotic spinal deformity: a biomechanical rationale for the clinical consequences and treatment of vertebral body compression frac- tures. J Spinal Disord Tech 2004;17:236-42.

20. Baroud G, Nemes J, Heini P, Steffen T. Load shift of the intervertebral disc after a vertebroplasty: a finite-element study. Eur Spine J 2003;12:421-6.

21. Lunt M, O'Neill TW, Felsenberg D, et al. Characteristics of a prevalent vertebral deformity predict subsequent vertebral fracture: results from the European Prospective Osteoporosis Study (EPOS). Bone 2003;33:505-13.

22. Kobayashi N, Numaguchi Y, Fuwa S, et al. Prophylactic vertebroplasty: cement injection into non-fractured vertebral bodies during percutaneous vertebroplasty. Acad Radiol 2009;16:136-43.

23. Chiang CK, Wang YH, Yang CY, Yang BD, Wang JL. Prophylactic vertebroplasty may reduce the risk of adjacent intact vertebra from fatigue injury: an ex vivo biomechanical study. Spine (Phila Pa 1976) 2009;34:356-64.

24. Kim SH, Kang HS, Choi JA, Ahn JM. Risk factors of new compression fractures in adjacent vertebrae after percutaneous vertebroplasty. Acta Radiol 2004;45:440-5.

25. Stricker K, Orler R, Yen K, Takala J, Luginbühl M. Severe hypercapnia due to pulmonary embolism of polymethylmethacrylate during vertebroplasty. Anesth Analg 2004; 98:1184-6.

26. Hulme PA, Krebs J, Ferguson SJ, Berlemann U. Vertebroplasty and kyphoplasty: a systematic review of 69 clinical studies. Spine (Phila Pa 1976) 2006;31:1983-2001.

27. Eck JC, Nachtigall D, Humphreys SC, Hodges SD. Comparison of vertebroplasty and balloon kyphoplasty for treatment of vertebral compression fractures: a meta-analysis of the literature. Spine J 2008;8:488-97. 